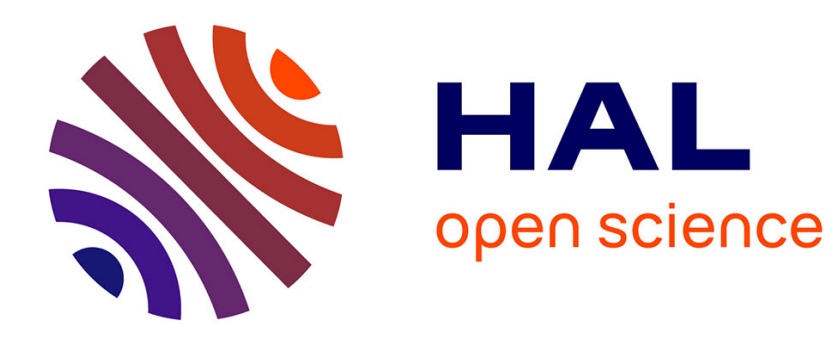

\title{
Velocities, Stresses and Vector Bundle Valued Chains
} Reuven Segev, Lior Falach

\section{To cite this version:}

Reuven Segev, Lior Falach. Velocities, Stresses and Vector Bundle Valued Chains. Journal of Elasticity, 2011, 10 (1-2), pp.187-206. hal-01060926

\section{HAL Id: hal-01060926 https://hal.science/hal-01060926}

Submitted on 4 Sep 2014

HAL is a multi-disciplinary open access archive for the deposit and dissemination of scientific research documents, whether they are published or not. The documents may come from teaching and research institutions in France or abroad, or from public or private research centers.
L'archive ouverte pluridisciplinaire HAL, est destinée au dépôt et à la diffusion de documents scientifiques de niveau recherche, publiés ou non, émanant des établissements d'enseignement et de recherche français ou étrangers, des laboratoires publics ou privés. 


\title{
Velocities, Stresses and Vector Bundle Valued Chains
}

\author{
Reuven Segev · Lior Falach
}

\begin{abstract}
A mathematical framework for the fundamental objects of continuum mechanics is presented. In the geometric setting of general differentiable manifolds, velocity fields over bodies, modeled as sections of a vector bundle $W$, are generalized using notions of homological integration theory such as flat chains and cochains. The class of bodies includes fractal sets whose irregular boundaries may have infinite measures. Stresses, initially modeled as smooth differential forms valued in the dual of the jet bundle of $W$, are generalized to cochains represented by $L^{\infty}$-sections whose weak divergences are also $L^{\infty}$. The divergence of a stress field, defined in an earlier work, is generalized to apply to stress cochains. The co-divergence of a velocity field is a weak form of the jet extension mapping and it is the counterpart of the boundary operator for real valued flat chains.
\end{abstract}

Keywords Continuum mechanics - Velocity - Stress - Vector valued - Vector bundle $\cdot$ Flat chains · Geometric integration theory - Geometric measure theory · de Rham currents · Divergence $\cdot$ Boundary operator

\section{Introduction}

This article is concerned with the mathematical setting of force and stress theory of continuum mechanics. On the one hand, the objective from the analytic point of view is to present a mathematical setting in which the smoothness or regularity properties required from bodies, velocity fields and stress fields may be relaxed and irregular bodies such as fractals may

R. Segev (西) · L. Falach

Department of Mechanical Engineering, Ben-Gurion University, P.O. Box 653, 84105 Beer-Sheva, Israel

e-mail: rsegev@bgu.ac.il

L. Falach

e-mail: falachl@bgu.ac.il 
be considered. As examples of recent works on continuum mechanics fundamentals for irregular bodies see $[5,24,25]$. On the other hand, from the geometric point of view, it is our objective to formulate the theory in the setting of general differentiable manifolds.

As early as 1957, Noll [16] defined a body in continuum mechanics as a differentiable manifold. Even in the case where the physical space is considered as a Euclidean space, if no natural reference state is given, a body does not have a natural Riemannian metric. Furthermore, it is quite a common practice to represent the internal state of a material point by additional parameters representing the microstructure or substructure (see, for example, [2, 15]). In cases where the internal variables do not represent geometric objects, for example, when they represent concentrations of various constituents, neither a natural Riemannian metric, nor a connection are available on the manifold of microstructure. (See, for example, $[3,12-14]$.) Formulations of continuum mechanics on general differentiable manifolds were presented in [20-23].

A convenient framework for the formulation of flux theory in continuum mechanics is H. Whitney's geometric integration theory [26]. Of particular importance is the theory of flat chains and cochains. A presentation of flux theory from the point of view of geometric integration theory was given in $[18,19]$. From the point of view of geometric integration theory, both a body and a potential field on it are represented using a single mathematical object, a chain. A flux is defined as a continuous linear functional on the space of chains and as such, duality theory implies that one obtains optimal spaces for fluxes and bodies such that the pairing between them is continuous. For example, various fractal sets whose boundaries are very irregular and of infinite measure (e.g., the von Koch snowflake or other examples in [1]) are admissible bodies if their measures (rather than the measures of the corresponding boundaries) are finite. Flat chain theory also defines continuous boundary operators so that a generalized version of Stokes' theorem is available.

Whitney's geometric integration theory is formulated for real-valued fluxes in a Euclidean space. In order to create a setting for continuum mechanics on manifolds, flat chain and cochain theory should be extended to vector bundle-valued fields on a manifold. Rather than extending Whitney's theory, we found it easier to adopt the approach by Federer and Fleming to the theory of flat chains and cochains. (See [6-8].) While Federer's approach is formulated in $\mathbb{R}^{n}$, it is based on de Rham's current theory [4] and as such, its generalization to manifolds is more natural. The drawback of this approach is the lack of geometric flavor afforded by Whitney's theory.

Thus, we use an extension of flat chain and cochain theory to sections of vector bundles on manifolds and achieve the same level of geometric generality as in [21-23]. A basic difference between the real-valued case and the vector-valued case that one has to overcome, is the unavailability of exterior derivatives. The divergence of stress fields, defined first in [22], serves as a partial replacement for the exterior derivative.

In Sect. 2 we review the main geometric objects in the case of smooth fields. These are the objects whose regularity properties we relax in the following sections. In particular, we review the notions of traction stresses, variational stresses and the divergence for a vector bundle over an $n$-dimensional manifold. Section 3 outlines the theory of real-valued flat chains and cochains in $\mathbb{R}^{n}$. In particular, we describe the Federer-Fleming approach to be used later. Section 4 introduces vector bundle-valued flat chains on manifolds and Sect. 5 represents velocity fields on irregular bodies and hypersurfaces as vector-valued flat chains. When velocity fields are regarded as sections of a general vector bundle, one cannot separate the derivative of the velocity field from the field itself and the exterior derivative is not defined. As a result, the derivative of a generalized velocity field is not a chain of a homogeneous degree, but rather, a combination of a chain of degree $n$ and a chain of degree 
$(n-1)$-a non-homogeneous chain. Non-homogeneous flat chains and their "boundaries"co-divergences-are considered in Sect. 6. Cochains, that model force and stress fields of relaxed smoothness, are considered in Section 7. Stresses may be as irregular as $L^{\infty}$-sections whose weak divergences are $L^{\infty}$, too.

The coboundary $\mathrm{d} X$ of a cochain $X$ is defined by $\mathrm{d} X(A)=X(\partial A)$ for every flat chain $A$. It is shown in geometric integration theory that $\mathrm{d} X$ is represented by an $L^{\infty}$-form which is the weak exterior derivative of the $L^{\infty}$-form representing $X$. In the setting of this work, this equation is generalized to $\operatorname{div} X_{S}(w)=X_{S}(\boldsymbol{\partial} w)$, where $\boldsymbol{\partial} w$ is the co-divergence of the velocity field $w, X_{S}$ is the vector-valued cochain represented by the variational stress $S$, and $\operatorname{div} X_{S}$ is the cochain represented by weak divergence of $S$.

\section{Flux and Stress Objects-the Smooth Setting}

\subsection{Body Forces and Velocity Fields}

We consider an $n$-dimensional oriented manifold $\mathscr{B}$ interpreted as the material manifold of continuum mechanics. As such, no Riemannian structure is assumed for $\mathscr{B}$. A vector bundle $\pi: W \rightarrow \mathscr{B}$ over $\mathscr{B}$ models the values of virtual velocities that the various material points may experience. For example, if a configuration is given as a section $e$ of a fiber bundle $\mu: \mathscr{E} \rightarrow \mathscr{B}$, so that $e(X) \in \mathscr{E}_{x}$ is the configuration of the point $x \in \mathscr{B}$, then $W_{x}$ may be thought of as the tangent space $T_{e(x)} \mathscr{E}_{x}$. An $n$-dimensional compact submanifold $B \subset \mathscr{B}$ which has a boundary may be thought of as a body. Sections of the form $w: \mathscr{B} \rightarrow W$ model global velocity fields of the material universe. The typical fiber of the vector bundle will be denoted by $\mathbf{W}$ and we set $\operatorname{dim} \mathbf{W}=m$. We will use $\left\{\left(U_{a}, \varphi_{a}, \Phi_{a}\right)\right\}$, to denote a vector bundle atlas so that for each $a, U_{a} \subset \mathscr{B}$ is open, $\varphi_{a}: U_{a} \rightarrow \mathbb{R}^{n}$ is a chart on $\mathscr{B}$ and $\Phi_{a}: \pi^{-1}\left(U_{a}\right) \rightarrow \varphi_{a}\left(U_{a}\right) \times \mathbb{R}^{m}$ is a local trivialization. (It is assumed that the vector bundle charts include base vectors for the typical fiber, hence, they take values in $\mathbb{R}^{n} \times \mathbb{R}^{m}$.) If $B \subset \mathscr{B}$ is a submanifold, we will write also $w: B \rightarrow W$ for a local section of the vector bundle without indicating that the co-domain of $w$ is actually $\left.W\right|_{B}$.

Natural integrands on $n$-dimensional manifolds are $n$-forms. Thus, a body force density $\beta$ is a field that once applied to a velocity field gives the density of the mechanical power in the body-an $n$-form. The value $\beta(x)$ at a point $x \in \mathscr{B}$ belongs to the space of linear mappings $L\left(W_{x}, \bigwedge^{n} T_{x}^{*} \mathscr{B}\right)$. Here, $\bigwedge^{n} T_{x}^{*} \mathscr{B}$, is the space of $n$-multilinear alternating forms so that $\beta(x)(w(x))\left(v_{1}, \ldots, v_{n}\right)$ is intuitively interpreted as the power produced by the body force $\beta$ for the virtual velocity field $w$ inside the infinitesimal simplex at $x$ defined by the vectors $v_{1}, \ldots, v_{n}$ (a tetrahedron in the 3-dimensional case). It follows that if $L\left(W, \bigwedge^{n} T^{*} \mathscr{B}\right)$ denotes the vector bundle of linear mappings, so that its fiber at $x \in \mathscr{B}$ is $L\left(W_{x}, \bigwedge^{n} T_{x}^{*} \mathscr{B}\right)$, a body force is a section $\beta: \mathscr{B} \rightarrow L\left(W, \bigwedge^{n} T^{*} \mathscr{B}\right)$. Using Riemann integration of forms, the total power produced by the body force for a virtual velocity field $w$ over a body $B$ is

$$
P=\int_{B} \beta(w) .
$$

Here, $\beta(w)$ is the $n$-form such that $\beta(w)(x)=\beta(x)(w(x))$. The expression for the power is a bi-linear functional in both $w$ and $\beta$. While physically, and as described above, it is convenient to think of generalized forces as operators acting on velocities to produce mechanical power, in most of this paper, specifically, Sects. 4-6, we emphasize the action of the local velocity field on the corresponding force fields over $\mathscr{B}$. Thus, we will regard the expression above as the action of a functional $T_{\langle w B\rangle}$ on the body force field so that $P=T_{\langle w B\rangle}(\beta)$. This 
functional, defined by the body $B$ and the virtual velocity field $w$ on it, is clearly linear in the body force field.

It is noted that by transposition, a body force field may be regarded as a vector-valued differential form. Given the section $\beta: \mathscr{B} \rightarrow L\left(W, \bigwedge^{n} T^{*} \mathscr{B}\right)$, there is a uniquely induced section $\beta^{\top}: \mathscr{B} \rightarrow \bigwedge^{n}\left(T \mathscr{B}, W^{*}\right)$, where an element $b \in \bigwedge^{n}\left(T_{x} \mathscr{B}, W_{x}^{*}\right)$ is an alternating, multilinear mapping $\left(T_{x} \mathscr{B}\right)^{n} \rightarrow W_{x}^{*}$. The $n$-form $\beta^{\top}$, valued in the dual bundle $W^{*}$, is defined by

$$
\beta^{\top}(x)\left(v_{1}, \ldots, v_{n}\right)(u)=\beta(x)(u)\left(v_{1}, \ldots, v_{n}\right)
$$

\subsection{Surface Forces and Velocity Fields on a Boundary of a Body}

Surface forces produce power densities over the boundaries of bodies. Consider a body $B$ with a smooth boundary $\partial B$. For a velocity field $u: \partial B \rightarrow W$, a surface force $\tau$ acts on $u$ to produce a power density-a real-valued $(n-1)$-form—over the boundary. For $y \in \partial B$, $\tau(y)(u(y))\left(v_{1}, \ldots, v_{n-1}\right)$, represents intuitively the power produced by the surface force $\tau$ for the velocity field $u$ inside the simplex defined by the vectors $v_{1}, \ldots, v_{n-1}$. Thus, $\tau$ is a section of $L\left(\left.W\right|_{\partial B}, \bigwedge^{n-1} T^{*} \partial B\right)$. It is noted that although this is not indicated in the notation, $\tau$ is defined only on $\partial B$ for the particular $B$ under consideration.

As in the case of body forces, the surface force $\tau$ induces a vector-valued form $\tau^{\top}$. The $(n-1)$-form $\tau^{\top}$, a section of $\bigwedge^{n-1}\left(T(\partial B),\left(\left.W\right|_{\partial B}\right)^{*}\right)$, is defined by

$$
\tau^{\top}(y)\left(v_{1}, \ldots, v_{n-1}\right)(u)=\tau(y)(u)\left(v_{1}, \ldots, v_{n-1}\right) .
$$

Let $D$ be an $(n-1)$-dimensional submanifold of $\partial B$. The total power produced by the surface force for the velocity field $u$ over $D$ is

$$
P=\int_{D} \tau(u)
$$

Again, the expression of the power is a bi-linear functional in both $u$ and $\tau$. In analogy with the case of body forces, we define the functional $T_{\langle u D\rangle}$ on the space of surface forces by

$$
T_{\langle u D\rangle}(\tau)=\int_{D} \tau(u)
$$

\subsection{Traction-stresses and Velocity Fields on Hypersurfaces}

While a surface force field is defined only on the boundary of a particular body $B$, a tractionstress field $\sigma$ gives the power density associated with a velocity field defined on an arbitrary $(n-1)$-dimensional hypersurface $D$ in $\mathscr{B}$. The appropriate object is a section

$$
\sigma: \mathscr{B} \rightarrow L\left(W, \bigwedge^{n-1} T^{*} \mathscr{B}\right)
$$

Thus, given a velocity field $w$, an $(n-1)$-real-valued form $\sigma(w)$ defined as $\sigma(w)(x)=$ $\sigma(x)(w(x))$ is induced in $\mathscr{B}$. For any collection of $n-1$ vectors $v_{1}, \ldots, v_{n-1}, \sigma(w)(x)\left(v_{1}\right.$, $\left.\ldots, v_{n-1}\right)$ is interpreted intuitively as the total power expanded in the hypersurface element defined by the vectors. Thus, given an oriented hypersurface $D \subset \mathscr{B}$, we may write the power expanded in $D$ as

$$
P=\int_{D} \sigma(w)
$$


The traction-stress induces a unique $W^{*}$-valued $(n-1)$-form $\sigma^{\top}: \mathscr{B} \rightarrow \bigwedge^{n-1}\left(T \mathscr{B}, W^{*}\right)$, by

$$
\sigma^{\top}(x)\left(v_{1}, \ldots, v_{n-1}\right)(u)=\sigma(x)(u)\left(v_{1}, \ldots, v_{n-1}\right) .
$$

Given a body $B$, a section $\sigma^{\top}$ of $\bigwedge^{n-1}\left(T B, W^{*}\right)$ may be restricted to the submanifold $\partial B \subset \mathscr{B}$ giving a section $\left.\sigma^{\top}\right|_{\partial B}$ of $\bigwedge^{n-1}\left(\left.T B\right|_{\partial B},\left.W^{*}\right|_{\partial B}\right)$. Moreover, the section $\left.\sigma^{\top}\right|_{\partial B}$ may be restricted to the subbundle $\left.T(\partial B) \subset T B\right|_{\partial B}$ to yield a section

$$
\rho_{\partial B}\left(\sigma^{\top}\right): \partial B \rightarrow \bigwedge^{n-1}\left(T(\partial B),\left.W^{*}\right|_{\partial B}\right) .
$$

In other words,

$$
\rho_{\partial B}\left(\sigma^{\top}\right)(y)\left(v_{1}, \ldots, v_{n-1}\right)=\sigma^{\top}(y)\left(v_{1}, \ldots, v_{n-1}\right) \in W_{y}^{*}
$$

for all $y \in \partial B, v_{1}, \ldots, v_{n-1} \in T_{y}(\partial B)$. The $W^{*}$-valued $(n-1)$-form $\rho_{\partial B}\left(\sigma^{\top}\right)$ on $\partial B$ induces by transposition a surface force field $\tau$, i.e.,

$$
\tau^{\top}=\rho_{\partial B}\left(\sigma^{\top}\right)
$$

The last equation is a generalization of the Cauchy formula for the compatibility of a traction-stress field $\sigma$ with a surface force field $\tau$ on $\partial B$.

A traction-stress is represented locally in the form

$$
\sum_{i, \alpha} \sigma_{\alpha 1 \ldots \hat{\imath} \ldots n} e^{\alpha} \otimes \mathrm{d} x^{1} \wedge \cdots \wedge \widehat{\mathrm{d} x^{i}} \wedge \cdots \wedge \mathrm{d} x^{n},
$$

where a superimposed "hat" indicates the omission of the corresponding term and $e^{\alpha}$ denotes an element of the local dual basis of the fibers $W_{x}$.

Remark 1 In an earlier work, [22], in order to distinguish them from the variational stresses to be described below, we referred to the traction-stresses as Cauchy stresses. That terminology may be misleading because the traction-stresses generalize both the Cauchy stresses and the Piola-Kirchhoff stresses. In view of their basic property reflected in (11), the term "traction-stresses" seems to reflect their nature better.

\subsection{The Variational Stress}

While the traction stress was characterized by the traction force fields it induces on hypersurfaces, the variational stress, which is a different geometric object for the general setting considered here, is characterized by its pairing with the derivative of a differentiable velocity field. As the derivative of a vector field on a general vector bundle is not an invariant object, one has to replace it by the jet of the velocity section whose local representatives contain the components of the vector field itself along with the partial derivatives of these components.

For a vector bundle $W$, we use the notation $J^{r}(W) \rightarrow W$ for the $r$-th jet bundle (see [17]). We recall that for $x \in \mathscr{B}, J^{1}(W)_{x}$ is isomorphic with $W_{x} \times L\left(T_{x} \mathscr{B}, W_{x}\right)$. The jet extension mapping $j^{1}: C^{1}(W) \rightarrow C^{0}\left(J^{1}(W)\right)$ assigns a section $j^{1}(w)$ of the jet bundle to a vector field $w$. If $w$ is represented locally by the components $\left(x^{i}, w^{\alpha}\right)$, then, $j^{1}(w)$ is represented locally by the components $\left(x^{i}, w^{\alpha}, w_{, j}^{\gamma}\right), \alpha, \gamma=1, \ldots, m, i, j=1, \ldots, n$, where a subscripted comma indicates partial differentiation. Thus, in order to apply the setting of 
forms on $\mathscr{B}$ valued in a vector bundle $W$ to the current situation, one has to replace $W$ by $J^{1}(W)$ which has the structure of a vector bundle both over $\mathscr{B}$ and over $W$, where the projection of the latter is given locally as $\left(x^{j}, u^{\alpha}, v_{i}^{\gamma}\right) \mapsto\left(x^{j}, u^{\alpha}\right)$. To produce power densities, a variational stress $S$ is defined therefore as a section of $L\left(J^{1}(W), \bigwedge^{n} T^{*} \mathscr{B}\right)$. For a body $B$ and a section $A$ of the jet bundle over $B$, we have a pairing

$$
\langle A, S\rangle=\int_{B} S(A) .
$$

It is noted that a section $A$ of the jet bundle need not be compatible in the sense that for a point $x \in \mathscr{B}$ and local representatives $\left(x^{j}, A^{\alpha}\left(x^{k}\right), A_{i}^{\alpha}\left(x^{k}\right)\right)$, one need not have necessarily $A_{i}^{\alpha}=A_{, i}^{\alpha}$. A variational stress is represented locally in the form $\left(x^{j}, R_{\alpha 1 \ldots n}\left(x^{k}\right), S_{\gamma 1 \ldots n}^{i}\left(x^{k}\right)\right)$ so that the $n$-form $S(A)$ is represented as

$$
\left(\sum_{\alpha} R_{\alpha 1 \ldots n} A^{\alpha}+\sum_{\alpha, i} S_{\alpha 1 \ldots n}^{i} A_{i}^{\alpha}\right) \mathrm{d} x^{1} \wedge \cdots \wedge \mathrm{d} x^{n} .
$$

Consider the case where the section $A$ is compatible, i.e., $A=j^{1}(w)$ for some vector field $w$, $A^{\alpha}\left(x^{k}\right)=w^{\alpha}\left(x^{k}\right)$ and $A_{i}^{\gamma}\left(x^{k}\right)=w_{, i}^{\gamma}\left(x^{k}\right)$. Then, the single component of the power density $S\left(j^{1}(w)\right)$ assumes the form

$$
\sum_{\alpha} R_{\alpha 1 \ldots n} w^{\alpha}+\sum_{\alpha, i} S_{\alpha 1 \ldots n}^{i} w_{, i}^{\alpha}=\sum_{\alpha, i}\left(R_{\alpha 1 \ldots n}-S_{\alpha 1 \ldots n, i}^{i}\right) w^{\alpha}+\sum_{\alpha, i}\left(S_{\alpha 1 \ldots n}^{i} w^{\alpha}\right)_{, i} .
$$

If $x^{i}=x^{i}\left(x^{i^{\prime}}\right), u^{\alpha}=\sum_{\alpha^{\prime}} \Psi_{\alpha^{\prime}}^{\alpha}\left(x^{i}\right) u^{\alpha^{\prime}}$ is a transformation of coordinates, the corresponding transformations for the components of $S$ are

$$
\begin{aligned}
R_{\alpha^{\prime} 1^{\prime} \ldots n^{\prime}} & =\left(\sum_{\alpha} R_{\alpha 1 \ldots n} \Psi_{\alpha^{\prime}}^{\alpha}+\sum_{\alpha, i} S_{\alpha 1 \ldots n}^{i} \Psi_{\alpha^{\prime}, i}^{\alpha}\right) \operatorname{det}\left(x_{, q^{\prime}}^{p}\right) \\
S_{\alpha^{\prime} 1^{\prime} \ldots n^{\prime}}^{i^{\prime}} & =\sum_{\alpha, i} S_{\alpha 1 \ldots n}^{i} \Psi_{\alpha^{\prime}}^{\alpha} x_{, i}^{i^{\prime}} \operatorname{det}\left(x_{, q^{\prime}}^{p}\right) .
\end{aligned}
$$

In analogy with (2), the object $S^{\top}$ is a section of $\bigwedge^{n}\left(T \mathscr{B}, J^{1}(W)^{*}\right)$.

\subsection{The Traction-stress Induced by a Variational Stress}

While the geometric object representing a variational stress is different than that representing a traction-stress, there is an invariant surjective, linear vector bundle morphism

$$
p_{\sigma}: L\left(J^{1}(W), \bigwedge^{n} T^{*} \mathscr{B}\right) \rightarrow L\left(W, \bigwedge^{n-1} T^{*} \mathscr{B}\right)
$$

that associates with every variational stress $S$ a traction-stress $\sigma=p_{\sigma} \circ S$ which we also write as $p_{\sigma}(S)$. Using the transposition isomorphisms $\bigwedge^{n}\left(T \mathscr{B}, J^{1}(W)^{*}\right) \cong L\left(J^{1}(W)\right.$, $\left.\bigwedge^{n} T^{*} \mathscr{B}\right)$ and $\bigwedge^{n-1}\left(T \mathscr{B}, W^{*}\right) \cong L\left(W, \bigwedge^{n-1} T^{*} \mathscr{B}\right)$, one may also regard $p_{\sigma}$ as a vector bundle morphism

$$
\bigwedge^{n}\left(T \mathscr{B}, J^{1}(W)^{*}\right) \rightarrow \bigwedge^{n-1}\left(T \mathscr{B}, W^{*}\right)
$$


Let $S$ be given locally by $\left(R_{\alpha 1 \ldots n}, S_{\gamma 1 \ldots n}^{i}\right)$, then, $\sigma=p_{\sigma} \circ S$ is given locally by

$$
\sigma_{\alpha 1 \ldots \hat{\imath} \ldots n}=(-1)^{i-1} S_{\alpha 1 \ldots n}^{i} .
$$

It can be shown (see [22]) that this local expression indeed represents an invariant mapping. For a section $S$ of $L\left(J^{1}(W), \bigwedge^{n} T^{*} \mathscr{B}\right), p_{\sigma}(S)$ may be regarded as the symbol of the linear differential operator on $W$ given by $S$ as in [17].

\subsection{The Divergence of a Variational Stress}

The divergence, defined in [22], is a linear differential operator

$$
\operatorname{div}: C^{1}\left(\bigwedge^{n}\left(T \mathscr{B},\left(J^{1}(W)\right)^{*}\right)\right) \rightarrow C^{0}\left(\bigwedge^{n}\left(T \mathscr{B}, W^{*}\right)\right) .
$$

Using the transposition isomorphism the divergence may be regarded also as a mapping

$$
C^{1}\left(L\left(J^{1}(W), \bigwedge^{n} T^{*} \mathscr{B}\right)\right) \rightarrow C^{0}\left(L\left(W, \bigwedge^{n} T^{*} \mathscr{B}\right)\right) .
$$

It is defined by

$$
\operatorname{div} S(w)=\mathrm{d}\left(p_{\sigma}(S)(w)\right)-S\left(j^{1}(w)\right),
$$

for every differentiable vector field $w$, where the transposition isomorphism is used again. To present the local expression for $\operatorname{div} S$ we first note that if $\sigma=p_{\sigma}(S)$, then $\mathrm{d}(\sigma(w))$ is represented locally by

$$
\begin{aligned}
& \sum_{\alpha, i} \mathrm{~d}\left(\sigma_{\alpha 1 \ldots \widehat{\imath} \ldots n} w^{\alpha}\right) \wedge \mathrm{d} x^{1} \wedge \cdots \wedge \widehat{\mathrm{d} x^{i}} \wedge \cdots \wedge \mathrm{d} x^{n} \\
& =\sum_{\alpha, i}\left(\sigma_{\alpha 1 \ldots \widehat{\imath} \ldots n} w^{\alpha}\right)_{, i} \mathrm{~d} x^{i} \wedge \mathrm{d} x^{1} \wedge \cdots \wedge \widehat{\mathrm{d} x^{i}} \wedge \cdots \wedge \mathrm{d} x^{n}, \\
& =\sum_{\alpha, i}\left(\sigma_{\alpha 1 \ldots \widehat{\ldots} \ldots n} w^{\alpha}\right)_{, i}(-1)^{i-1} \mathrm{~d} x^{1} \wedge \cdots \wedge \mathrm{d} x^{n}, \\
& =\sum_{\alpha, i}\left(S_{\alpha 1 \ldots n}^{i} w^{\alpha}\right)_{, i} \mathrm{~d} x^{1} \wedge \cdots \wedge \mathrm{d} x^{n} .
\end{aligned}
$$

The local expression for $\operatorname{div} S$ is therefore

$$
\sum_{\alpha, i}\left(S_{\alpha 1 \ldots n, i}^{i}-R_{\alpha 1 \ldots n}\right) e^{\alpha} \otimes \mathrm{d} x^{1} \wedge \cdots \wedge \mathrm{d} x^{n} .
$$

For a given variational stress, the definition of the divergence together with Stokes' theorem imply that

$$
-\int_{B} \operatorname{div} S(w)+\int_{\partial B} p_{\sigma}(S)(w)=\int_{B} S\left(j^{1}(w)\right) .
$$

Setting $\beta=-\operatorname{div} S$,

$$
\int_{B} \beta(w)+\int_{\partial B} p_{\sigma}(S)(w)=\int_{B} S\left(j^{1}(w)\right)
$$


a generalization of the principle of virtual work in continuum mechanics.

It is noted that in the case where the variational stress has a compact support in the interior of $B$, the boundary integral above vanishes and one has

$$
\int_{B} S\left(j^{1}(w)\right)=-\int_{B} \operatorname{div} S(w)
$$

\section{Real-valued Flat Chains and Co-chains in Euclidean Spaces}

\subsection{Flat Chains and Flat Co-chains from the Point of View of Whitney's Geometric Integration Theory}

Whitney's geometric integration theory, [26], provides a convenient setting for the formulation of flux theory in Euclidean spaces (see [19]). Choosing geometric integration theory as a setting for continuum theory of fluxes, one obtains an analytically optimal formulation as fluxes are modeled as linear functionals - cochains-on the Banach space of chains. By optimality, we mean that the combination of a collection of bodies and a collection of fluxes, is in some sense the largest. Bodies may be as irregular as some fractal sets. Flux fields and integration of fluxes over the boundaries are defined for such irregular bodies. The differential balance law is valid where the exterior derivative of the flux field is uniquely defined almost everywhere.

Geometric integration theory has an appealing physical interpretation. Bodies are built using oriented simplices as building blocks. A simplex $s$ may be multiplied by a real number $a$. The number multiplying a simplex may be interpreted as a uniform potential (e.g., an electrostatic potential) over that simplex. The formal sum $\sum_{k} a_{k} s_{k}$ of such products, where the simplices may be made disjoint by further subdivision, is then regarded as a step function describing the piecewise constant potential field over the union of the simplices. The collection of these piecewise uniform fields is then completed relative to the flat norm so that the complete space, the space of flat chains, contains smooth fields on manifolds but also, for example, $L^{1}$-fields and bodies with fractal boundaries. If we consider $n$-simplices in an $n$-dimensional Euclidean space $E$, an $n$-cochain may be interpreted as the operation of calculating the rate of change of the total of the energy corresponding to the given potential. Thus, for the example of an electric potential, a cochain will be an operator that assigns the time derivative of the total electrostatic energy to a given chain representing a potential field over some body. It is a consequence of Whitney's theory, that the operator may be represented as an integration operator of a density, the rate of change of the electric charge density, over this chain.

The boundary $\partial B$ of a flat chain $B$ is well defined. It is a flat $(n-1)$-chain and it includes both the boundary value of the potential field as well as its gradient. Thus, an $(n-1)$-cochain $T$, represents physically both the energy flux through the boundary of the corresponding body due to the flux of charge, and the rate of change of energy due to flow of current in a non-uniform potential field. As expected, the representation theorem for cochains implies that an $(n-1)$-cochain may be represented by a flux field.

\subsection{Federer's Approach to Real-valued Flat Chains and Cochains in Euclidean Spaces}

In geometric measure theory, as in [6,7] or [8], an alternative equivalent approach to flat chain theory is presented. In the overview below and the rest of this paper, our notation is 
different than that of Federer in order to suit better the extension to general manifolds and vector-valued forms.

A flat $r$-chain is defined as an $r$-de Rham-current. It is recalled (see [4]) that an $r$-current on a manifold $\mathscr{M}$ is a continuous, linear functional $T$ on the space $C_{0}^{\infty}\left(\bigwedge^{r} T^{*} \mathscr{M}\right)$ of smooth $r$-forms of compact supports. By continuity, it is meant that if $\left(\theta_{k}\right)$ is a sequence of forms supported in a compact set $K$ contained in the domain $U$ of a chart, and the local representatives of $\theta_{k}$ as well as all their partial derivatives tend to zero uniformly on $U$, then $T\left(\theta_{k}\right) \rightarrow 0$. We also recall that the boundary $\partial T$ of a real-valued $r$-current $T$ is the $(r-1)$-current defined by duality as $\partial T(\omega)=T(\mathrm{~d} \omega)$. Since for any form $\theta, \mathrm{d}^{2} \theta=0$, one has $\partial^{2} T=0$.

Federer's flat chain theory considers currents in an open set $U \subset \mathbb{R}^{n}$. For the sake of simplicity, it is assumed here that we consider forms supported in a fixed compact set $K \subset U$. For a current $T$, set $|T|=\sup _{\theta}|T(\theta)|$ where the supremum is taken over all smooth forms $\theta$, supported in $K$, such that $\sup _{x \in U}|\theta(x)| \leq 1$. The magnitude $|\theta(x)|$ of the alternating tensor $\theta(x)$ is computed using the natural Euclidean structure of $\mathbb{R}^{n}$. The value $|T|$, which might be infinite, is referred to as the mass of the current. A current $T$ is said to be normal, if both $T$ and $\partial T$ are of finite mass.

The flat norm of a form $\theta$ is defined as

$$
\|\theta\|^{b}=\max \left\{\sup _{x \in U}|\theta(x)|, \sup _{x \in U}|\mathrm{~d} \theta(x)|\right\} .
$$

For a current $T$, define the flat norm by

$$
\|T\|^{b}=\sup _{\|\theta\|^{b} \leq 1} T(\theta) .
$$

Since $\mathrm{d}^{2} \theta=0$, one has $\|\partial T\|^{\mathrm{b}} \leq\|T\|^{\mathrm{b}}$. Thus, the boundary operator is bounded in the flat topology. In addition, as $\|\theta\|^{b} \geq \sup _{x}|\theta(x)|,\|T\|^{b} \leq|T|$, and it follows that if the mass of a current is finite so is its flat norm. In particular, a normal current has a finite flat norm. Flat chains are elements of the completion of the space of normal currents in the flat norm. The Banach space of flat $r$-chains, acting on forms in $U$ supported in $K$, will be denoted by $\mathbf{F}_{r, K}(U)$. Thus, the boundary operator

$$
\partial: \mathbf{F}_{r, K}(U) \rightarrow \mathbf{F}_{r-1, K}(U)
$$

is continuous.

Let $\xi$ be an $(n-r)$-form whose components are integrable. The $r$-current $T_{\langle\xi\rangle}$ induced by $\xi$ is defined, using Lebesgue integration of $n$-forms, as

$$
T_{\langle\xi\rangle}(\theta)=\int_{U} \xi \wedge \theta
$$

This definition applies also in the case of a differentiable manifold where local representatives of the form $\xi$ are required to be integrable (see $[4,10]$ ). In the case where $\xi$ is differentiable, for all smooth $(r-1)$-forms $\omega$ supported in $U$,

$$
\partial T_{\langle\xi\rangle}(\omega)=\int_{U} \xi \wedge \mathrm{d} \omega=(-1)^{(n-r+1)} \int_{U} \mathrm{~d} \xi \wedge \omega,
$$

where we used the identity $d(\xi \wedge \omega)=d \xi \wedge \omega+(-1)^{\text {degree } \xi} \xi \wedge d \omega$, and the fact that $\operatorname{supp} \omega \subset U$. It follows that in the case where $\xi$ is differentiable,

$$
\partial T_{\langle\xi\rangle}=(-1)^{n-r+1} T_{\langle\mathrm{d} \xi\rangle} .
$$


In [6, pp. 375-376], a representation theorem for flat chains in terms of forms is proved. Expressed using the notation adopted here, the theorem implies that for a flat $r$-chain $T$ and any $\delta>0$, there is an integrable $(n-r)$-form $\xi$ and an integrable $(n-r-1)$-form $\eta$ both of which are supported inside a set containing all points in $U$ whose distance from $K$ is smaller than $\delta$, such that

$$
T=T_{\langle\xi\rangle}+\partial T_{\langle\eta\rangle}, \quad \text { or equivalently, for all } \theta, \quad T(\theta)=\int_{U}(\xi \wedge \theta+\eta \wedge \mathrm{d} \theta) .
$$

In addition,

$$
\int_{U}(|\xi|+|\eta|)-\delta \leq\|T\|^{b} \leq \int_{U}(|\xi|+|\eta|) .
$$

Conversely, for any pair of forms $\xi$ and $\eta$ as above, the current $T$ defined by (34) is a flat chain. It is noted that if $T$ is represented by $\xi$ and $\eta$ as in (34) above, then,

$$
\partial T=\partial T_{\langle\xi\rangle}, \quad \text { or equivalently, for all } \omega, \quad \partial T(\omega)=\int_{U} \xi \wedge \mathrm{d} \omega .
$$

For a flat $n$-chain $T$, the representation simplifies to $T=T_{\langle\xi\rangle}$ so that flat chains are represented by $L^{1}, n$-forms.

A flat $r$-cochain is a linear functional on the space of flat chains that is bounded by the flat norm. As the boundary operator is continuous and linear on the spaces of chains, we have the dual coboundary operator

$$
\mathrm{d}=\partial^{*}: \mathbf{F}_{r, K}(U)^{*} \rightarrow \mathbf{F}_{r+1, K}(U)^{*}
$$

which extends the exterior derivative to cochains.

The representation theorem for flat chains in terms of $L^{1}$-forms implies the following representation of flat cochains. To every flat $r$-cochain $X$, there is an $r$-form $D_{X}$ and an $(r+1)$-form $D_{X}^{\prime}$ both of which are $L^{\infty}$. When $T$ is represented as $T=T_{\langle\xi\rangle}+\partial T_{\langle\eta\rangle}$, so that

$$
X(T)=X\left(T_{\langle\xi\rangle}\right)+X\left(\partial T_{\langle\eta\rangle}\right)
$$

one has

$$
X\left(T_{\langle\xi\rangle}\right)=\int_{U} \xi \wedge D_{X}, \quad \text { and } \quad X\left(\partial T_{\langle\eta\rangle}\right)=\int_{U} \eta \wedge D_{X}^{\prime}
$$

In addition,

$$
\mathrm{d} X(T)=X(\partial T)=X\left(\partial T_{\langle\xi\rangle}\right)=\int_{U} \xi \wedge D_{X}^{\prime} .
$$

We may conclude that $D_{X}^{\prime}$ represents the coboundary $\mathrm{d} X$ and this motivates the notation $D_{\mathrm{d} X}=D_{X}^{\prime}$. In addition, for all differentiable $\xi$,

$$
\begin{aligned}
\mathrm{d} X(T) & =X\left(\partial T_{\langle\xi\rangle}\right)=(-1)^{n-r+1} X\left(T_{\langle\mathrm{d} \xi\rangle}\right) \\
& =(-1)^{n-r+1} \int \mathrm{d} \xi \wedge D_{X}=\int_{U} \xi \wedge D_{\mathrm{d} X} .
\end{aligned}
$$

The second row in the last equation implies that $D_{\mathrm{d} X}$ is the weak exterior derivative of $D_{X}$, so that in the weak sense $D_{\mathrm{d} X}=\mathrm{d} D_{X}$. In the case where $\eta$ is differentiable, (38) assumes 
the form

$$
\begin{aligned}
X(T) & =X\left(T_{\langle\xi\rangle}\right)+(-1)^{n-r+1} X\left(T_{\langle\mathrm{d} \eta\rangle}\right), \\
& =\int_{U}\left(\xi+(-1)^{n-r+1} \mathrm{~d} \eta\right) \wedge D_{X} .
\end{aligned}
$$

The forms $D_{X}$ and $D_{\mathrm{d} X}$ that represent the cochain $X$ are unique up to inequality on sets of measure zero. See [9-11] for a different approach to the representation of cochains and for properties of $L^{\infty}$-forms with $L^{\infty}$-exterior derivatives on manifolds.

\section{Vector-valued Flat Chains on Manifolds}

\subsection{Vector-valued Forms and Linear Functionals}

In order that the theory of flat chains and cochains be suitable for force and stress theory in continuum mechanics, the forms considered should be vector-valued as described in Sect. 2. For an $n$-dimensional manifold $\mathscr{B}$ and a vector bundle $\pi: W \rightarrow \mathscr{B}$ whose typical fiber is $m$-dimensional, one may consider flat chains valued in $W$ by extending the theory of Federer and Fleming [6, 7] described above.

In a natural extension of the theory of de Rham currents, the test functions we consider are smooth sections $\theta$ of $\bigwedge^{r}\left(T \mathscr{B}, W^{*}\right) \cong \bigwedge^{r} T^{*} \mathscr{B} \otimes W^{*}$ that have compact supports. Here, the choice of values in $W^{*}$ rather than in $W$ is made for the sake of convenience. Let $\left\{\left(U_{a}, \varphi_{a}, \Phi_{a}\right)\right\}$, be a vector bundle atlas. A $W^{*}$-valued $r$-form $\theta$ is represented locally in the form

$$
\sum_{\alpha, i_{1}, \ldots, i_{r}} \theta_{\alpha i_{1} \ldots i_{r}} e^{\alpha} \otimes \mathrm{d} x^{i_{1}} \wedge \cdots \wedge \mathrm{d} x^{i_{r}}=\sum_{\alpha,(I)} \theta_{\alpha i_{1} \ldots i_{r}} e^{\alpha} \otimes \mathrm{d} x^{i_{1}} \wedge \cdots \wedge \mathrm{d} x^{i_{r}}
$$

Here, $i_{1}, \ldots, i_{r}=1, \ldots, n, i_{1}<i_{2}<\cdots<i_{r}$. Using multi-index notation (e.g., $I=$ $\left.\left(i_{1}, \ldots, i_{l}\right)\right)$, parentheses around multi-indices indicate that the indices are increasing (e.g., $\left.i_{1}<\cdots<i_{l}\right)$. For each $\alpha, \theta_{\alpha}=\sum_{(I)} \theta_{\alpha i_{1} \ldots i_{r}} \mathrm{~d} x^{i_{1}} \wedge \cdots \wedge \mathrm{d} x^{i_{r}}$ is a real-valued $r$-form in $U_{a}$. Using the notation $\theta^{(a)}=\left.\theta\right|_{U_{a}}$, in the case where we wish to emphasize that $\theta_{\alpha}$ or $\theta_{\alpha i_{1} \ldots i_{r}}$ are the local representatives under the $\left(U_{a}, \varphi_{a}, \Phi_{a}\right)$-chart, we will write $\theta_{\alpha}^{(a)}$ or $\theta_{\alpha i_{1} \ldots i_{r}}^{(a)}$, respectively.

Consider a partition of unity $\left\{\left(\eta_{a}, K_{a}\right)\right\}$ subordinate to the vector bundle atlas $\left\{\left(U_{a}, \varphi_{a}\right.\right.$, $\left.\Phi_{a}\right)$ \}. For each $a, K_{a}$ is a compact subset of $U_{a}, \eta_{a}$ is a smooth real-valued function defined on $\mathscr{B}$ supported in $K_{a}$ and $\sum_{a} \eta_{a}=1$. For a vector-valued form $\theta$, the form $\eta_{a} \theta$ is supported in $K_{a}$ and evidently $\left(\eta_{a} \theta\right)_{\alpha}^{(a)}=\left.\eta_{a}\right|_{U_{a}} \theta_{\alpha}^{(a)}$. For any vector-valued form $\theta$ one clearly has $\theta=\sum_{a} \eta_{a} \theta$.

We consider linear functionals $T$ on the space of smooth vector-valued forms supported in a compact set $K \subset \mathscr{B}$. This simplification causes only little loss of generality. For the given vector bundle atlas, letting $\left.T\right|_{K_{a}}$ be the restriction of $T$ to forms that are compactly supported in $K_{a}$, one has

$$
\begin{aligned}
T(\theta) & =T\left(\sum_{a} \eta_{a} \theta\right)=\left.\sum_{a} T\right|_{K_{a}}\left(\eta_{a} \theta\right)=T\left(\sum_{a} \eta_{a} \theta^{(a)}\right) \\
& =\sum_{a} T \circ \Phi_{a}^{-1}\left(\Phi_{a} \circ\left(\eta_{a} \theta^{(a)}\right)\right)=\sum_{a} T_{a}\left(\Phi_{a} \circ\left(\eta_{a} \theta^{(a)}\right)\right)
\end{aligned}
$$




$$
=\sum_{a, \alpha} T_{a}^{\alpha}\left(\eta_{a} \theta_{\alpha}^{(a)}\right)=\sum_{a, \alpha,(I)} T_{a}^{\alpha i_{1} \ldots i_{r}}\left(\eta_{a} \theta_{\alpha i_{1} \ldots i_{r}}^{(a)}\right)
$$

where $T_{a}=\left.T\right|_{K_{a}} \circ \Phi_{a}^{-1}$ and its components are $T_{a}^{\alpha}$-real-valued currents in $\mathbb{R}^{n}$. Here and elsewhere, for the sake of simplifying the notation, we did not indicate the zero extension of $\theta^{(a)}$ from $U_{a}$ to $\mathscr{B}$ needed in order to multiply it by $\eta_{a}$ and apply $T$ to the product. One concludes that it is sufficient to determine $T$ by the action of its local representatives on real-valued forms compactly supported in the various $U_{a}$.

\subsection{The Flat Topology and Vector-valued Flat Chains on Manifolds}

In analogy with the $C^{r}$-topology on the space of differential forms, the flat topology is defined using a vector bundle atlas and a partition of unity $\mathscr{A}=\left\{\left(U_{a}, \varphi_{a}, \Phi_{a}, \eta_{a}, K_{a}\right)\right\}$ by

$$
\|\theta\|_{\mathscr{A}}^{\mathrm{b}}=\max _{a, \alpha, I, J}\left\{\sup _{x \in U_{a}}\left|\theta_{\alpha I}^{(a)} \eta_{a}(x)\right|, \sup _{x \in U_{a}}\left|\left[\mathrm{~d}\left(\theta_{\alpha I}^{(a)} \eta_{a}\right)\right]_{J}(x)\right|\right\} .
$$

It is recalled that for two manifolds $\mathscr{M}$ and $\mathscr{M}^{\prime}$ and an embedding $F: \mathscr{M} \rightarrow \mathscr{M}^{\prime}$, the induced pullback mapping,

$$
F^{*}:\left.\bigwedge^{r} T^{*} \mathscr{M}^{\prime}\right|_{\text {Image } F} \rightarrow \bigwedge^{r} T^{*} \mathscr{M}
$$

satisfies the identity $F^{*}(\mathrm{~d} \theta)=\mathrm{d} F^{*}(\theta)$. When applied to the local representatives of differential forms and to transformations of coordinates, this identity implies that the local representative of $\mathrm{d} \theta$ under a chart $\left(U_{a}, \varphi_{a}, \Phi_{a}\right)$ depends only on the representative of $\mathrm{d} \theta$ under an overlapping chart $\left(U_{b}, \varphi_{b}, \Phi_{b}\right)$ (and does not depend, for example, on partial derivatives of the representatives of $\theta$ that are not included in the exterior derivative). We also note that for $n$-forms the exterior derivative vanishes so that $\|\theta\|_{\mathscr{A}}^{\mathrm{b}}=\max _{a, \alpha, I}\left\{\sup _{x \in U_{a}}\left|\theta_{\alpha I}^{(a)} \eta_{a}(x)\right|\right\}$.

While $\|\theta\|_{\mathscr{A}}^{\mathrm{b}}$ depends on $\mathscr{A}$, the topology induced by this norm is independent of the choices of atlas and partition of unity. We will refer to it as the flat topology. In analogy with the topology defined on the space of smooth forms for the definition of currents as above, one could define a linear functional $T$ to be continuous for the flat topology if for the case of a sequence $\left(\theta_{k}\right)$ whose elements are supported in a compact set $K_{0}$ contained in the domain $U$ of a chart, such that the local representatives of $\theta_{k}$ and $\mathrm{d} \theta_{k}$ tend to zero uniformly in $K_{0}$, $T\left(\theta_{k}\right) \rightarrow 0$. In addition, the norm

$$
\max _{a, \alpha, I, J}\left\{\sup _{x \in U_{a}}\left|\theta_{\alpha I}^{(a)}(x)\right|, \sup _{x \in U_{a}}\left|\left[\mathrm{~d}\left(\theta_{\alpha I}^{(a)}\right)\right]_{J}(x)\right|\right\}
$$

is equivalent to $\|\theta\|_{\mathscr{A}}^{b}(c f .[17$, pp. 10-14]).

When the space of smooth $W^{*}$-valued $r$-forms is equipped with the flat topology, we will denote it by $\mathscr{D}_{r}^{b}\left(W^{*}\right)$.

A vector-valued flat $r$-chain on the manifold $\mathscr{B}$ is a linear functional $T$ on $\mathscr{D}_{r}^{\mathrm{b}}\left(W^{*}\right)$ whose local representatives $T_{a}^{\alpha}$ as in (44) are (real-valued) flat chains in $\mathbb{R}^{n}$. It follows that for a given $\mathscr{A}$ as above, a vector-valued flat chain $T$ on $\mathscr{B}$ is continuous relative to the $\|\cdot\|_{\mathscr{A}}^{b}$-norm. We conclude that vector-valued flat chains are continuous relative to the flat topology for vector-valued forms on $\mathscr{B}$. Naturally, we refer to $T$ as a $W$-valued flat $r$-chain if it is dual to the space of $r$-forms-valued in $W^{*}$. 


\subsection{Fields Over Real-valued Flat Chains}

We now consider a special case of vector-valued flat $r$-chains on manifolds. Let $w$ be a differentiable section of $L\left(W^{*}, \bigwedge^{p} T^{*} \mathscr{B}\right) \cong \bigwedge^{p} T^{*} \mathscr{B} \otimes W$, for $p \leq r$. Thus, $w(x)$ may be represented in the form

$$
w(x)=\sum_{l} \omega^{l} \otimes v_{l}
$$

where $\omega^{l} \in \bigwedge^{p} T_{x}^{*} \mathscr{B}, v_{l} \in W_{x}$. In addition, let $\theta$ be a section of $L\left(W, \bigwedge^{r-p} T^{*} \mathscr{B}\right) \cong$ $\bigwedge^{r-p} T^{*} \mathscr{B} \otimes W^{*}$, so that $\theta(x)$ may be represented in the form

$$
\theta(x)=\sum_{s} \zeta^{s} \otimes \gamma^{s}
$$

where $\zeta^{s} \in \bigwedge^{r-p} T_{x}^{*} \mathscr{B}, \gamma^{s} \in W_{x}^{*}$. We now use the notation

$$
\theta(x) \dot{\wedge} w(x)=\sum_{l, s} \gamma^{s}\left(v_{l}\right) \zeta^{s} \wedge \omega^{l},
$$

so that $\theta \dot{\lambda} w$ is a real-valued $r$-form, $(\theta \dot{\lambda} w)(x)=\theta(x) \dot{\lambda} w(x)$.

Let $w$ be represented locally as

$$
\sum_{\alpha,(J)} w_{j_{1} \ldots j_{p}}^{\alpha} e_{\alpha} \otimes \mathrm{d} x^{j_{1}} \wedge \cdots \wedge \mathrm{d} x^{j_{p}}
$$

and let $\theta$ be represented locally as

$$
\sum_{\alpha,(I)} \theta_{\alpha i_{1} \ldots i_{l}} e^{\alpha} \otimes \mathrm{d} x^{i_{1}} \wedge \cdots \wedge \mathrm{d} x^{i_{l}},
$$

where $l+p=r$. Then, $\theta \dot{\wedge} w$ is represented by

$$
\sum_{\alpha,(I),(J),(K)} \epsilon_{k_{1} \ldots \ldots k_{r}}^{i_{1} \ldots i_{l} j_{1} \ldots j_{p}} \theta_{\alpha i_{1} \ldots i_{l}} w_{j_{1} \ldots j_{p}}^{\alpha} \mathrm{d} x^{k_{1}} \wedge \cdots \wedge \mathrm{d} x^{k_{r}},
$$

where $\epsilon_{k_{1} \ldots \ldots j_{r} \ldots j_{r}}^{i_{1} \ldots j_{p}}$ denotes the alternating symbol.

Let $C$ be a real-valued flat $r$-chain on $\mathscr{B}$, so that its local representatives are real-valued flat chains on the images of the various charts. For a differentiable section $w$ of $W$ (regarded as a vector-valued 0 -form) we define the $W$-valued $r$-flat chain $T_{\langle w C\rangle}=w C$ by

$$
T_{\langle w C\rangle}(\theta)=w C(\theta)=C(\theta \dot{\wedge} w) .
$$

Writing the norms for the local representatives of $\theta \dot{\lambda} w$, one realizes that $w C$ is indeed a flat chain. In fact, the local representatives of $w C$ are real-valued flat $r$-chains in an open subset of $\mathbb{R}^{n}$ of the form $\phi C$, where $\phi$ is a bounded function with bounded Lipschitz constant as in [26, p. 208]. Accordingly, we will refer to $T_{\langle w C\rangle}=w C$ as the product of the field $w$ with the real-valued $r$-flat chain $C$.

Remark 2 For a real-valued flat $r$-chain, or in general, for a real-valued de Rham $r$-current, $T$, the boundary operator, defined by $\partial T(\theta)=T(\mathrm{~d} \theta)$, is linear and continuous. However, for vector bundle valued forms on manifolds, the exterior derivative is not defined. Hence, the boundaries of vector-valued flat chains on manifolds are not defined in the general case. 


\section{Velocity Fields as Vector-valued Chains}

5.1 Generalized Velocity Fields on Bodies as Vector-valued Flat $n$-chains

Using the terminology of vector-valued flat chains on manifolds, we can now extend the pairing of body forces and velocity fields so that we admit generalized velocity fields ("generalized" in the distributional sense) modeled as flat chains.

Let $w$ be a vector field in $\mathscr{B}$ whose local representatives relative to the atlas and partition of unity $\mathscr{A}=\left\{\left(U_{a}, \varphi_{a}, \Phi_{a}, \eta_{a}, K_{a}\right)\right\}$ are $L^{1}$-functions. In such a case, $w$ is referred to as an integrable section. For any body force field $\beta$, a smooth section of $L\left(W, \bigwedge^{n} T^{*} \mathscr{B}\right)$, set

$$
T_{\langle w\rangle}(\beta)=\int_{\mathscr{B}} \beta(w) .
$$

Using the vector bundle atlas and partition of unity, one has

$$
\begin{aligned}
T_{\langle w\rangle}(\beta) & =\int_{\mathscr{B}} \sum_{a} \eta_{a} \beta(w), \\
& =\sum_{a}\left(\int_{\varphi_{a}\left(K_{a}\right)} \beta_{\alpha 1 \ldots n}\left(\eta_{a} \circ \varphi_{a}^{-1}\right) w^{\alpha}\right) .
\end{aligned}
$$

Observing that the $\left(\eta_{a} \circ \varphi_{a}^{-1}\right) w^{\alpha}$ are integrable functions supported in $\varphi_{a}\left(K_{\alpha}\right)$, it follows from the representation theorem for flat chains that for any chart $a$ and each $\alpha=1, \ldots, m$, $\left(\eta_{a} \circ \varphi_{a}^{-1}\right) w^{\alpha}$ represents a flat $n$-chain in $\mathbb{R}^{n}$. Thus, $T_{\langle w\rangle}$ is a $W$-valued flat $n$-chain on $\mathscr{B}$.

In the particular case where $B$ is a measurable set and $w$ is an $L^{\infty}$-vector field in $\mathscr{B}$, the vector field $w \chi_{B}$, where $\chi_{B}$ is the characteristic function of $B$, is integrable. The $W$-valued flat $n$-chain induced by $w \chi_{B}$ is denoted by $T_{\langle w B\rangle}=w B$. It is the product of the vector field $w$ by the flat chain $B$ induced by $\chi_{B}$. Clearly,

$$
T_{\langle w B\rangle}(\beta)=w B(\beta)=\int_{B} \beta(w) .
$$

As a generalization, one could also replace the characteristic function $\chi_{B}$ by any other integrable real-valued function on $\mathscr{B}$.

5.2 Generalized Velocity Fields as Vector-valued Flat $(n-1)$-chains on $\partial B$

Assume we are given a particular body manifold $B \subset \mathscr{B}$. Considering surface forces on $\partial B$, i.e., $W^{*}$-valued $(n-1)$-forms on the compact manifold $\partial B$, one observes that the only difference from the case of body forces is the dimension of the manifold. Thus, an atlas and a partition of unity should apply to the restriction $\left.W\right|_{\partial B} \rightarrow \partial B$ of $W$.

Let $u$ be an integrable section of $\left.W\right|_{\partial B}$. The linear operator $T_{\langle u\rangle}$ operating on surface forces and defined by

$$
T_{\langle u\rangle}(\tau)=\int_{\partial B} \tau(u)
$$

is represented locally by integration of integrable functions. It follows that $T_{\langle u\rangle}$ is a $\left.W\right|_{\partial B^{-}}$ valued, $(n-1)$-chain on $\partial B$. 
5.3 Generalized Velocity Fields on Hypersurfaces as Vector-valued Flat $(n-1)$-chains in $\mathscr{B}$

The pairing

$$
P=\int_{D} \sigma(w)
$$

of a traction-stress field $\sigma$ and the restriction of a vector field $w$ to an $(n-1)$-dimensional submanifold $D$ may be generalized to an action of a $W$-valued flat $(n-1)$-chain on tractionstress fields. Such flat chains represent fields over generalized surfaces. For example, various fractal surfaces such as the boundary of the von Koch snowflake may be represented as realvalued flat chains. In addition, the class of flat chains also contains continuous chains which are different from the intuitive image of a vector field over a hypersurface. For example, if $w$ is an $L^{1}, W$-valued 1-form, then, for any traction-stress $\sigma, \sigma \dot{\wedge} w$ is an $n$-form and $T_{\langle w\rangle}$ defined by

$$
T_{\langle w\rangle}(\sigma)=\int_{\mathscr{B}} \sigma \dot{\wedge} w
$$

is a $W$-valued flat $(n-1)$-chain. This chain is a generalization of the continuous real-valued chains in [26]. For the real-valued case in $\mathbb{R}^{n}$, it is convenient to interpret $\sigma$ as the charge flux vector field and $w$ as the electric field. However, this interpretation does not carry over to the vector bundle case.

A particular situation of interest is the case where the $W$-valued flat $(n-1)$-chain $T$ is given by a Lipschitz vector field $w$ on $\mathscr{B}$, regarded as a $W$-valued zero-form, and a realvalued $(n-1)$-chain $C$. As in Sect. 4.3, one may set $T_{\langle w C\rangle}=w C$, where,

$$
w C(\sigma)=C(\sigma \dot{\wedge} w)=C(\sigma(w))
$$

since $w$ is a zero-form. In the terminology of [26], this may be written as

$$
w C(\sigma)=T_{\langle w C\rangle}(\sigma)=\int_{C} \sigma(w) .
$$

In the case where the real-valued $(n-1)$-chain $C$ represents a generalized hypersurface, e.g., the fractal boundary of a body having a finite volume, the action $w C(\sigma)$ generalizes the expression for the power of the induced surface force for the given velocity field $w$.

\subsection{Jet Bundle-valued Flat $n$-chains}

Jet bundle-valued flat $n$-chains act on variational stress fields to generalize the pairing $\langle A, S\rangle=\int_{B} S(A)$, for a body $B$ and a section $A$ of the jet bundle over $B$. In particular, for the case where $A=j^{1}(w)$ for a differentiable vector field $w$ in $W,\langle A, S\rangle=\int_{B} S\left(j^{1}(w)\right)$.

Let $A$ be an $L^{1}$-section of the jet bundle $J^{1}(W)$. Then, the components of its local representatives are $L^{1}$-functions in open subsets of $\mathbb{R}^{n}$ that represent a collection of real-valued flat chains according to Federer's representation theorem. Thus, we have a representation of jet bundle-valued flat $n$-chains in the form

$$
T_{\langle A\rangle}(S)=\int_{\mathscr{B}} S(A)
$$

for integrable sections of the jet bundle. 
A different topology on the space of smooth variational stresses, to be introduced below, will give a more suitable framework for considerations of flat chains that act on variational stresses.

\section{Non-homogeneous Flat Chains}

\subsection{The Variational Stress as a Non-homogeneous $(n-1)$-form}

In Sect. 5.4 we treated variational stress fields as jet bundle-valued $n$-forms and the corresponding chains are continuous relative to the sup-norm of the components of the local representatives. The topology considered here is a variation of the flat topology for $(n-1)$-forms and we will refer to it as the flat topology for variational stress fields. Let $\mathscr{A}=\left\{\left(U_{a}, \varphi_{a}, \Phi_{a}, \eta_{a}, K_{a}\right)\right\}$ be a vector bundle atlas and a subordinate partition of unity. For a smooth variational stress field $S$, set

$$
\|S\|_{\mathscr{A}}^{\odot}=\max \left\{\|S\|_{\mathscr{A}}^{\mathrm{b}},\|\operatorname{div} S\|_{\mathscr{A}}^{\mathrm{b}}\right\},
$$

where, for the norms on the right-hand side, $S$ and $\operatorname{div} S$ are considered as vector-valued $n$ forms. (Note that since $S$ and $\operatorname{div} S$ are $n$-forms, no exterior derivative of the local representatives is included in their flat norms relative to an atlas and partition of unity.) Thus, in the flat topology, $S \rightarrow 0$, if all local representatives, $R_{\alpha 1 \ldots n}, S_{\alpha 1 \ldots n}^{i}$, and $\sum_{i} S_{\alpha 1 \ldots n, i}^{i}-R_{\alpha 1 \ldots n}$, tend to zero uniformly on all charts. For $\alpha=1, \ldots, m$, consider the components of the local representatives, the real-valued $n$-forms $\sigma_{\alpha}=p_{\sigma}(S)_{\alpha}=\sum_{i} \sigma_{\alpha 1 \ldots \hat{\imath} \ldots n} \mathrm{~d} x^{1} \wedge \cdots \wedge \widehat{\mathrm{d} x^{i}} \wedge \cdots \wedge \mathrm{d} x^{n}$ so that $\sigma_{\alpha 1 \ldots \hat{i} \ldots n}=(-1)^{i-1} S_{\alpha 1 \ldots n}^{i}$. One has,

$$
\begin{aligned}
\mathrm{d} \sigma_{\alpha} & =\sum_{i} \mathrm{~d}\left(\sigma_{\alpha 1 \ldots \hat{\imath} \ldots n} \mathrm{~d} x^{1} \wedge \cdots \wedge \widehat{\mathrm{d} x^{i}} \wedge \cdots \wedge \mathrm{d} x^{n}\right) \\
& =\sum_{i} \sigma_{\alpha 1 \ldots \hat{\imath} \ldots n, i} \mathrm{~d} x^{i} \wedge \mathrm{d} x^{1} \wedge \cdots \wedge \widehat{\mathrm{d} x^{i}} \wedge \cdots \wedge \mathrm{d} x^{n}, \\
& =\sum_{i} \sigma_{\alpha 1 \ldots \widehat{I} \ldots n, i}(-1)^{i-1} \mathrm{~d} x^{1} \wedge \cdots \wedge \mathrm{d} x^{n}, \\
& =\sum_{i} S_{\alpha 1 \ldots n, i}^{k} \mathrm{~d} x^{1} \wedge \cdots \wedge \mathrm{d} x^{n} .
\end{aligned}
$$

It follows that $\max \left\{\|S\|_{\mathscr{A}}^{\mathrm{b}},\left\|p_{\sigma}(S)\right\|_{\mathscr{A}}^{\mathrm{b}}\right\}$, where $p_{\sigma}(S)$ is considered as a $W^{*}$-valued $(n-1)$ form, generates a topology equivalent to $\|S\|_{\mathscr{A}}^{\odot}$.

Henceforth, we will use $\mathscr{D}^{\circ}\left(J^{1}(W)^{*}\right)$ to denote the topological vector space $C_{0}^{\infty}\left(L\left(J^{1}(W), \bigwedge^{n} T^{*} \mathscr{B}\right)\right)$ equipped with the topology induced by the $\mathcal{Y}$-norm as above. In accordance with Sect. 4.2, where we use the flat topology for $n$-forms on the same set of sections (as in (45)), we will denote the resulting topological vector space by $\mathscr{D}_{n}^{b}\left(J^{1}(W)^{*}\right)$.

Consider the mapping

$$
(l, \operatorname{div}): \mathscr{D}^{\bigcirc}\left(J^{1}(W)^{*}\right) \rightarrow \mathscr{D}_{n}^{b}\left(J^{1}(W)^{*}\right) \times \mathscr{D}_{n}^{\mathrm{b}}\left(W^{*}\right),
$$

where $\iota$ is the identity mapping and the topology on $\mathscr{D}_{n}^{b}\left(J^{1}(W)^{*}\right) \times \mathscr{D}_{n}^{b}\left(W^{*}\right)$ is induced by a norm of the form

$$
\|(\omega, \zeta)\|_{\mathscr{A}}=\max \left\{\|\omega\|_{\mathscr{A}}^{\mathrm{b}},\|\zeta\|_{\mathscr{A}}^{\mathrm{b}}\right\} .
$$


We observe that $(l$, div) is linear, injective, and a homeomorphism onto its image.

\subsection{Non-homogeneous Vector-valued Flat Chains and Velocity Fields}

Let $\left(T_{1}, T_{2}\right)$ be a linear functional on $\mathscr{D}_{n}^{\mathrm{b}}\left(J^{1}(W)^{*}\right) \times \mathscr{D}_{n}^{\mathrm{b}}\left(W^{*}\right)$ so that $\left(T_{1}, T_{2}\right)(\omega, \zeta)=$ $T_{1}(\omega)+T_{2}(\zeta)$, where $T_{1}$ and $T_{2}$ are flat $n$-chains valued in the corresponding vector bundles. By their definition in Sect. 4.2, $T_{1}$ and $T_{2}$ are represented locally by real-valued flat $n$-chains valued in $\mathbb{R}^{n}$. Since by Federer's representation theorem, real-valued flat $n$-chains in $\mathbb{R}^{n}$ are given in terms of $L^{1}, n$-forms, there are $L^{1}$-sections $d_{1}$ and $d_{2}$ of $J^{1}(W)$ and $W$, respectively, such that

$$
\left(T_{1}, T_{2}\right)(\omega, \zeta)=\int_{\mathscr{B}} \omega\left(d_{1}\right)+\int_{\mathscr{B}} \zeta\left(d_{2}\right) .
$$

Given $T_{1}$ and $T_{2}$ as above, a linear functional $T$ defined on $\mathscr{D}^{\ominus}\left(J^{1}(W)^{*}\right)$ of the form $T=(l, \operatorname{div}) \circ\left(T_{1}, T_{2}\right)$ will be referred to as a non-homogeneous vector-valued flat chain. Clearly, a non-homogeneous vector-valued flat chain is a continuous operator and may be represented in the form

$$
T(S)=\int_{\mathscr{B}} S\left(d_{1}\right)+\int_{\mathscr{B}} \operatorname{div} S\left(d_{2}\right),
$$

for some integrable sections $d_{1}$ and $d_{2}$ as above.

The Banachable space of non-homogeneous flat chains, which is isomorphic to $L^{1}\left(J^{1}(W)\right) \times L^{1}(W)$, will be denoted by $G\left(J^{1}(W)\right)$.

It is noted that in the case where $d_{2}$ is a differentiable section, it follows from the definition of the divergence and the fact that $S$ is compactly supported that

$$
\begin{aligned}
\int_{\mathscr{B}} \operatorname{div} S\left(d_{2}\right) & =\int_{\mathscr{B}} \mathrm{d}\left(p_{\sigma}(S)\left(d_{2}\right)\right)-\int_{\mathscr{B}} S\left(j^{1}\left(d_{2}\right)\right), \\
& \left.=\int_{\partial(\operatorname{supp} S)} p_{\sigma}(S)\left(d_{2}\right)\right)-\int_{\mathscr{B}} S\left(j^{1}\left(d_{2}\right)\right) \\
& =-\int_{\mathscr{B}} S\left(j^{1}\left(d_{2}\right)\right) .
\end{aligned}
$$

As a result, for the case of a differentiable $d_{2}$, the representation of the non-homogeneous flat chain assumes the form

$$
T(S)=\int_{\mathscr{B}} S\left(d_{1}-j^{1}\left(d_{2}\right)\right) .
$$

It is noted that a section of the jet bundle need not be the jet of a section of $W$. Thus, in analogy with classical continuum mechanics where one can apply the stress field to a tensor field that is not necessarily compatible, i.e., not necessarily the gradient of a velocity field, in the term $S\left(d_{1}\right), d_{1}$ need not be the jet, or a weak form of a jet, of a vector field. This clearly does not hold for the term $S\left(j^{1}\left(d_{2}\right)\right)$.

\subsection{The Co-divergence}

The basic property of the $\odot$-topology on the collection of variational stresses is that the divergence operator

$$
\operatorname{div}: \mathscr{D}^{\bigcirc}\left(J^{1}(W)^{*}\right) \rightarrow \mathscr{D}_{n}^{b}\left(W^{*}\right)
$$


is continuous. Recalling that a $W$-valued flat $n$-chain, a bounded linear functional on $\mathscr{D}_{n}^{b}\left(W^{*}\right)$, is of the form

$$
T_{\langle w\rangle}(\beta)=\int_{\mathscr{B}} \beta(w),
$$

for an integrable section $w$ of $W$, set

$$
\partial T_{\langle w\rangle}=\operatorname{div}^{*}\left(T_{\langle w\rangle}\right)=T_{\langle w\rangle} \circ \operatorname{div} .
$$

Then, $\partial T_{\langle w\rangle}$, which we refer to as the co-divergence of $T_{\langle w\rangle}$-a generalization of the boundary of a current-is a continuous linear functional on $\mathscr{D}^{\mathcal{O}}\left(J^{1}(W)^{*}\right)$. Specifically,

$$
\boldsymbol{\partial} T_{\langle w\rangle}(S)=\int_{\mathscr{B}} \operatorname{div} S(w) .
$$

In fact, comparing the last equation with the representation in (68), we note that $\partial T_{\langle w\rangle}$ is represented by $d_{1}=0$ and $d_{2}=w$.

In the particular case where $w$ is a differentiable velocity field,

$$
\partial T_{\langle w\rangle}(S)=-\int_{\mathscr{B}} S\left(j^{1}(w)\right) .
$$

Thus, the co-divergence operator may be described as the weak jet extension of the velocity field.

Consider the case where $B$ is a subset of $\mathscr{B}$ such that the characteristic function $\chi_{B}$ is integrable. If $w$ is a differentiable vector field on $\mathscr{B}$, then,

$$
\begin{aligned}
\partial T_{\langle w B\rangle}(S) & =\int_{B} \operatorname{div} S(w), \\
& =\int_{B} \mathrm{~d}\left(p_{\sigma}(S)(w)\right)-\int_{B} S\left(j^{1}(w)\right), \\
& =\int_{\partial B} p_{\sigma}(S)(w)-\int_{B} S\left(j^{1}(w)\right) .
\end{aligned}
$$

\section{Loads and Stresses as Vector-valued Cochains}

Once velocity fields are extended to $W$-valued flat $n$-chains, one may generalize smooth body force fields to cochains. Let $\beta$ be a $W^{*}$-valued $n$-form such that the components of its local representatives are $L^{\infty}$. Then, each component $\beta_{\alpha 1 \ldots n}$ is an $L^{\infty}$-form in an open subset $V_{a}$ of $\mathbb{R}^{n}$. A vector-valued flat $n$-chain is represented by a vector field $w$ whose components $w^{\alpha}$ are $L^{1}$-functions (or zero-forms) in the various $\varphi_{a}\left(U_{a}\right)$. It follows that the $L^{\infty}$-form $\beta$ represents a cochain $X_{\beta}$ in the form

$$
X_{\beta}\left(T_{\langle w\rangle}\right)=\int_{\mathscr{B}} \beta(w) .
$$

This is a trivial implication of the representation theorem for flat cochains as for an $n$-chain, the representing form $\eta$, as in (39), vanishes. The pairing of body forces and velocities in (1) has been generalized thus to velocities that are $L^{1}$ and body forces that are $L^{\infty}$. 
Analogically, for a body $B \subset \mathscr{B}$ having a smooth boundary, the paring of surface forces and boundary velocity fields as in (4) is generalized to the action of an $(n-1)$-cochain $Y_{\tau}$ on $\partial B$, represented by the $L^{\infty},(n-1)$-form $\tau$, on an $L^{1}$-vector field $u$ representing a flat $(n-1)$-chain in the form

$$
Y_{\tau}\left(T_{\langle u\rangle}\right)=\int_{\partial B} \tau(u) .
$$

Similarly, smooth traction stresses are generalized to $W^{*}$-valued $(n-1)$-forms $\sigma$ whose local representatives $\sigma_{\alpha}$ are $L^{\infty}$ and such that the weak exterior derivatives $\mathrm{d} \sigma_{\alpha}$ are also $L^{\infty}$. In [9-11] it is shown for the real-valued case that such forms are locally flat in the sense of [26]. We will simply use the term "flat forms" for the vector-valued case considered here. It is noted that for such a vector-valued form $\sigma$, whose corresponding cochain is $X_{\sigma}$, the coboundary is not defined as the exterior derivative of the traction stress cannot be defined invariantly.

Generalizing variational stresses to non-homogeneous $(n-1)$-cochains, we consider variational stresses $S$ whose components are $L^{\infty}$ and for which the components of the corresponding $\operatorname{div} S$ are also $L^{\infty}$. It is noted that by the definition of the divergence, for such a variational stress field $S$, the corresponding traction stress $\sigma=p_{\sigma}(S)$ is a flat vector-valued $(n-1)$-form. Thus, we will refer to $S$ as a non-homogeneous flat form. Observing Equation (68), it is clear that in case $S$ is a flat form, the pairing

$$
\langle T, S\rangle=\int_{\mathscr{B}} S\left(d_{1}\right)+\int_{\mathscr{B}} \operatorname{div} S\left(d_{2}\right)
$$

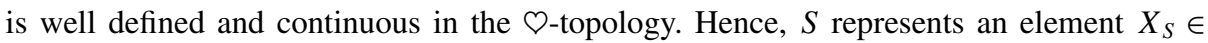
$G\left(J^{1}(W)\right)^{*}$.

From the definition of $S$ as a flat form, it is clear that $S \mapsto \operatorname{div} S$ is a continuous mapping into the space of $W^{*}$-valued $n$-cochains. Thus, the divergence is extended to a continuous co-co-divergence operator, for which we retain the same notation and the generalization of (25) is written as

$$
\operatorname{div} X_{S}(w)=X_{S}(\partial w)
$$

for each non-homogeneous flat chain $w$.

Acknowledgement This work was partially supported by the Paul Ivanier Center for Robotics Research and Production Management at Ben-Gurion University.

\section{References}

1. Barnsley, M.: Fractals Everywhere. Academic Press, New York (1988)

2. Capriz, G.: Continua with Microstructure. Springer, Berlin (1989)

3. Capriz, G., Mariano, P.: Symmetries and Hamiltonian formalism for complex materials. J. Elast. 72, 57-70 (2003)

4. de Rham, G.: Differentiable Manifolds. Springer, Berlin (1984)

5. Degiovanni, M., Marzocchi, A., Musesti, A.: Cauchy fluxes associated with tensor fields having divergence measure. Arch. Ration. Mech. Anal. 147, 197-223 (1999)

6. Federer, H.: Geometric Measure Theory. Springer, Berlin (1969)

7. Federer, H., Fleming, W.: Normal and integral currents. Ann. Math. 72(3), 458-520 (1960)

8. Giaquinta, M., Modica, G., Soucek, J.: Cartesian Currents in the Calculus of Variations I. Springer, Berlin (1998)

9. Gol'dshtein, V., Kuz'minow, V., Shvedov, I.: Integration of differential forms of the classes $\mathscr{W}_{p, q}^{*}$. Sib. Mat. Zh. 23, 63-79 (1982). English translation published by Plenum 
10. Gol'dshtein, V., Kuz'minow, V., Shvedov, I.: Differential forms on Lipschitz manifolds. Sib. Mat. Zh. 23, 16-30 (1982). English translation published by Plenum

11. Gol'dshtein, V., Kuz'minow, V., Shvedov, I.: Wolfe's theorem for differential forms of classes $\mathscr{W}_{p, q}^{*}$. Sib. Mat. Zh. 24, 31-42 (1983). English translation published by Plenum

12. Mariano, P.: Multifield theories in mechanics of solids. Adv. Appl. Mech. 38, 1-93 (2002)

13. Mariano, P.: Cracks in complex bodies: covariance of tip balances. J. Nonlinear Sci. 18, 99-141 (2008)

14. Mariano, P., Modica, G.: Ground states in complex bodies. ESAIM Control Optim. Calc. Var. 15, 377$402(2009)$

15. Mermin, N.: The topological theory of defects in ordered media. Rev. Mod. Phys. 51, 591-646 (1979)

16. Noll, W.: The foundation of classical mechanics in light of recent advances in continuum mechanics. In: The Axiomatic Method, with Special Reference to Geometry and Physics. North-Holland, Amsterdam (1959)

17. Palais, R.S.: Foundations of Global Non-Linear Analysis. Benjamin, Elmsford (1968)

18. Rodnay, G.: Cauchy's flux theorem in light of Whitney's geometric integration theory. Ph.D. thesis, Ben-Gurion University of the Negev (2002)

19. Rodnay, G., Segev, R.: Cauchy's flux theorem in light of geometric integration theory. J. Elast. 71, 183203 (2003)

20. Segev, R.: Forces and the existence of stresses in invariant continuum mechanics. J. Math. Phys. 27, 163-170 (1986)

21. Segev, R.: The geometry of Cauchy's fluxes. Arch. Ration. Mech. Anal. 154, 183-198 (2000)

22. Segev, R.: Metric-independent analysis of the stress-energy tensor. J. Math. Phys. 43, 3220-3231 (2002)

23. Segev, R., Rodnay, G.: Cauchy's theorem on manifolds. J. Elast. 56(2), 129-144 (1999)

24. Silhavy, M.: Cauchy's stress theorem for stresses represented by measures. Contin. Mech. Thermodyn. 20, 75-96 (2008)

25. Silhavy, M.: The divergence theorem for divergence measure vectorfields on sets with fractal boundaries. Math. Mech. Solids 14, 445-455 (2009)

26. Whitney, H.: Geometric Integration Theory. Princeton University Press, Princeton (1957) 